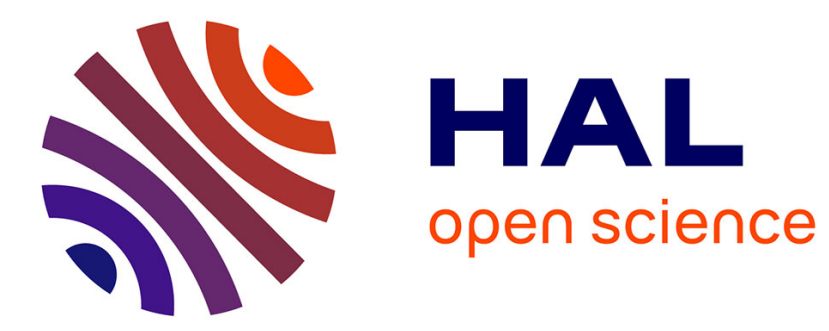

\title{
A multi-step solution algorithm for Maxwell boundary integral equations applied to low-frequency electromagnetic testing of conductive objects
}

Audrey Vigneron, Edouard Demaldent, Marc Bonnet

\section{- To cite this version:}

Audrey Vigneron, Edouard Demaldent, Marc Bonnet. A multi-step solution algorithm for Maxwell boundary integral equations applied to low-frequency electromagnetic testing of conductive objects. IEEE Transactions on Magnetics, 2016, 52, pp.7005208. 10.1109/TMAG.2016.2584018 . hal01336835

\section{HAL Id: hal-01336835 \\ https://hal.science/hal-01336835}

Submitted on 23 Jun 2016

HAL is a multi-disciplinary open access archive for the deposit and dissemination of scientific research documents, whether they are published or not. The documents may come from teaching and research institutions in France or abroad, or from public or private research centers.
L'archive ouverte pluridisciplinaire HAL, est destinée au dépôt et à la diffusion de documents scientifiques de niveau recherche, publiés ou non, émanant des établissements d'enseignement et de recherche français ou étrangers, des laboratoires publics ou privés. 


\title{
A multi-step solution algorithm for Maxwell boundary integral equations applied to low-frequency electromagnetic testing of conductive objects
}

\author{
Audrey Vigneron ${ }^{1}$, Edouard Demaldent ${ }^{1}$, Marc Bonnet ${ }^{2}$ \\ ${ }^{1}$ CEA, LIST, Saclay, 91191 Gif-sur-Yvette, France \\ 2 POEMS (UMR 7231 CNRS-INRIA-ENSTA), ENSTA, 828, boulevard des Maréchaux, 91762 Palaiseau Cedex, France
}

\begin{abstract}
We consider the solution, using boundary elements (BE), of the surface integral equation system arising in electromagnetic testing of conducting bodies, with emphasis on situations such that $o(1) \leq \sqrt{\omega \varepsilon_{0} / \sigma} \leq O(1), L \sqrt{\omega \sigma \mu_{0}}=O(1)$ which includes in particular the case of eddy current testing) and assuming $L \omega \sqrt{\varepsilon_{0} \mu_{0}} \leq 2 \pi$, i.e. low-frequency conditions ( $L$ : diameter of conducting body). Earlier approaches for dielectric objects at low frequencies are not applicable in the present context. After showing that a simple normalization of the BE system significantly improves its conditioning, we propose a multi-step solution method based on block SOR iterations, which facilitates the use of direct solvers and converges within a few iterations for the considered range of physical parameters. This novel, albeit simple, treatment allows to perform eddy current-type analyses using standard Maxwell SIE formulations, avoiding the adverse consequences of ill-conditioning for low frequencies and high conductivities. Its performance and limitations are studied on three numerical examples involfing low frequencies and high conductivities.
\end{abstract}

Index Terms-Maxwell equations, Electromagnetic testing, Eddy currents, low frequency, surface integral equations, boundary element method.

\section{INTRODUCTION}

W E consider the modelling of electromagnetic testing of objects characterized by their diameter $L$, conductivity $\sigma$, dielectric permittivity $\varepsilon^{\mathrm{d}}$ and magnetic permeability $\mu=\mu_{r} \mu_{0}$, for a wide range of values of $L, \sigma$ and angular frequency $\omega=2 \pi f$ ( $f$ : prescribed frequency). Testing is deemed to be in the low-frequency regime insofar as $L \leq \lambda_{0}$ will always be assumed, where $\lambda_{0}:=2 \pi /\left(\omega \sqrt{\varepsilon_{0} \mu_{0}}\right)$ is the wavelength in the medium, treated as vacuum, surrounding the conducting object being tested.

In particular, applications of eddy current non-destructive testing (ECNDT) [1], and more generally the eddy current (EC) regime, correspond to $\gamma \ll 1$ and $\xi=\mathcal{O}(1)$, in terms of the non-dimensional parameters

$$
\gamma:=\sqrt{\omega \varepsilon_{0} / \sigma}, \quad \xi:=L \sqrt{\omega \sigma \mu_{0}} .
$$

The wavenumbers $\kappa_{0}$ in vacuum and $\kappa_{1}$ in the conducting medium are then linked to $\gamma, \xi$ through

$$
\kappa_{0} L=\gamma \xi, \quad \kappa_{1} L=\mu_{r} \xi \sqrt{\left(\mathrm{i}+\varepsilon_{r}^{\mathrm{d}} \gamma^{2}\right)}
$$

As an example of typical ECNDT conditions, testing a part of conductivity $\sigma=10^{6} \mathrm{~S} / \mathrm{m}$ and size $L=10^{-2} \mathrm{~m}$ at a $10^{5} \mathrm{~Hz}$ frequency corresponds to $\gamma \simeq 2.35 \times 10^{-6}$ and $\xi \simeq 8.88$. Testing is sometimes performed at lower frequencies for magnetic and highly conducting media (e.g. $f=10^{2} \mathrm{~Hz}$, $\sigma=10^{7} \mathrm{~S} / \mathrm{m}$ and $\mu_{r}=10^{2}$, yielding $\gamma \simeq 2.35 \times 10^{-8}$ and $\xi \simeq 8.88 \times 10^{-1}$ ). Other electromagnetic testing configurations that are outside the EC regime while remaining in lowfrequency conditions exist, such as microwave testing for media of moderate conductivity (e.g. composite media [2] with $f=10^{9} \mathrm{~Hz}, \sigma=10^{2} \mathrm{~S} / \mathrm{m}, L=10^{-2} \mathrm{~m}$, for which $\gamma \simeq 2.35 \times 10^{-2}, \xi \simeq 8.88$ ) or terahertz testing. Moreover, still

Corresponding author: E. Demaldent (email: edouard.demaldent@cea.fr). other conditions of electromagnetic testing, involving weakly conducting media of large characteristic size, occur e.g. in applications of induction tomography in geophysics [3] (e.g. $f=10^{5} \mathrm{~Hz}, \sigma=10^{-2} \mathrm{~S} / \mathrm{m}$ and $L=10^{3} \mathrm{~m}$, for which $\gamma \simeq 2.35 \times 10^{-2}, \xi \simeq 8.88$ ). These considerations underline the usefulness of numerical electromagnetic simulation methods that perform well over the whole range of physical parameters such that $o(1) \leq \gamma \leq O(1)$ while $\xi=O(1)$ and are therefore applicable to the modeling of all of the above-mentioned testing situations.

Objects undergoing testing are here assumed to have homogeneous (or piecewise-homogeneous) properties, which allows modelling methods based on surface integral equation (SIE) formulations (see e.g. [4,5] for SIE-based EC modelling). For (e.g. geophysical) applications involving low frequencies and low conductivities, SIE-based methods may perform poorly due to ill-conditioning resulting from low frequency breakdown [6,7]. A partial remedy consists in applying a Helmholtz-Hodge decomposition to the surface unknowns involved in the SIE formulation of the transmission problem, whereby the usual $H_{\mathrm{div}}$-conforming edge-based basis functions defining the boundary element (BE) approximation space are converted into solenoidal (loop) and non-solenoidal (tree) functions $[7,8]$. The resulting BE system remains very illconditioned due to the inhomogeneous low-frequency behavior of the various submatrices induced by the loop-tree decomposition. The condition number of the global BE system may exceed $10^{20}$ at low frequencies, entailing serious accuracy and robustness issues even if using a LU-based direct solver. By contrast, each submatrix has a homogeneous low-frequency behavior, allowing the introduction of normalization matrices (even though this is not sufficient for ensuring reliable performance of iterative solvers). Such treatments usually assume the 

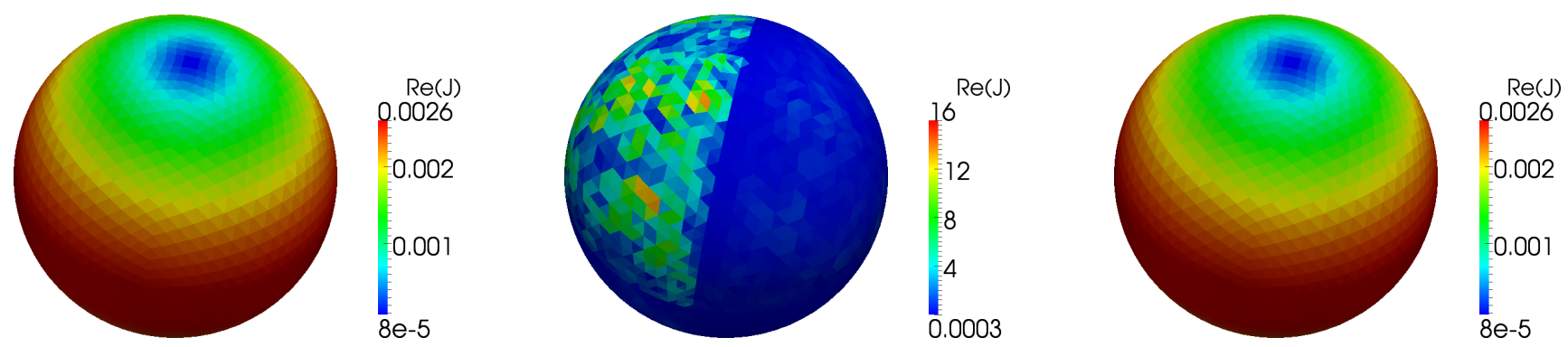

Fig. 1. Scattering of a plane wave by a conducting sphere, EC regime: real part of current density $J$ (in $A / \mathrm{m}^{2}$ ), with $\varepsilon_{r}^{\mathrm{d}}=1, \xi=0.1$ and $\gamma \approx 7.5 \times 10^{-4}$ (left), $\gamma \approx 7.5 \times 10^{-7}$ without (middle) and with (right) loop-tree decomposition. Left and right solutions are very similar, both cases being near the EC limit $\gamma \rightarrow 0, \xi=O(1)$.

wavenumber $\kappa$ to behave like $\kappa=O(\omega)$ at low frequencies.

More recently, the loop-tree decomposition was employed on highly conducting objects $\left(\sigma \gg \omega \varepsilon^{\mathrm{d}}\right.$, i.e. $\left.\varepsilon_{r}^{\mathrm{d}} \gamma^{2} \ll 1\right)$, whose low-frequency behavior obeys the EC approximation [9]. In such cases, the usual low-frequency normalization methods are no longer applicable as $\kappa_{1} \sim \mu_{r} \xi \sqrt{\mathrm{i}}=\delta^{-1} \sqrt{2 \mathrm{i}} \neq O(\omega)$ (typical values for the skin depth $\delta=\sqrt{2 /(\omega \sigma \mu)}$ being $\delta=$ $\left.O\left(10^{-3}\right) \mathrm{m}\right)$. As illustrated in Fig. 1 on a numerical example in EC regime involving a conducting sphere illuminated by a plane wave at frequencies either $f_{1}=1 \mathrm{MHz}$ or $f_{2}=1 \mathrm{~Hz}$ (with physical parameters otherwise such that $\varepsilon_{r}^{\mathrm{d}}=1, \xi=0.1$, and $\gamma \approx 7.5 \times 10^{-4}$ or $\gamma \approx 7.5 \times 10^{-7}$ for frequencies $f_{1}$ or $f_{2}$ ), using the loop-tree decomposition is a prerequisite for avoiding corrupted solutions when $\gamma$ is very low.

In this article, we first show that applying a natural normalization to the global BE system yields acceptable condition numbers that are very insensitive to the physical parameters for the range of interest $o(1) \leq \gamma \leq O(1), \xi=O(1)$. Then, we propose a multi-step algorithm based on block SOR iterations applied to the $4 \times 4$ block partition of the BE influence matrix naturally induced by the loop-tree decomposition of the electric and magnetic surface current densities, which are the primary BE unknowns. This method, together with its demonstration and discussion on representative examples, constitutes our main intended contribution. It extends the applicability of direct solvers as they are now used blockwise, and is shown to converge within few iterations for the considered range of physical parameters. As a result, it allows to perform ECtype analyses using standard Maxwell SIE formulations while avoiding the adverse consequences of ill-conditioning at low $\gamma$. A similar, albeit simpler, multi-step algorithm based on block Gauss-Seidel (GS) iterations on a $2 \times 2$ block partition of the BE matrix has been proposed in [10] for applications to dielectric objects. The present method improves on [10] in generality and flexibility, and is better suited to the simulation of electromagnetic testing in the EC regime.

This article is organized as follows. The proposed modifications of the loop-tree-decomposed BE system are presented in Section II, and subsequently demonstrated in Section III on three example problems under conditions corresponding to the EC regime. Factors influencing computational efficiency, and directions for future work, are discussed in Section IV.

\section{MOdified FORMULATIONS OF PMCHWT SYSTEM}

Electromagnetic testing involves a transmission problem whereby a three-dimensional bounded conducting object (or a set thereof) with complex permittivity $\varepsilon_{1}=\varepsilon^{\mathrm{d}}-\mathrm{i} \sigma / \omega$, surrounded by vacuum filling the unbounded surrounding space, is excited by given electric and magnetic fields (solving Maxwell's equations with $(\varepsilon, \mu)=\left(\varepsilon_{0}, \mu_{0}\right)$ in $\left.\mathbb{R}^{3}\right)$. The transmission problem is assumed to obey the well-known PMCHWT surface integral equation (SIE) system, whose primary unknowns are current densities $J$ (electric) and $M$ (magnetic) on the interface $\Gamma$ separating the conductor(s) and the vacuum. Following an approach previously used for low-frequency scattering by dielectric bodies [7], we first apply a loop-tree decomposition to the boundary element (BE) approximation space (here generated by RWG or rooftop basis functions). The resulting finite-dimensional linear system of equations can be written, in compact form, as

$$
\begin{aligned}
& Z X=Y \\
& \text { with }[Z]_{I J}=Z^{I J},\{X\}_{J}=X^{J},\{Y\}_{I}=Y^{I},
\end{aligned}
$$

where $I, J=1, \ldots, 4$ correspond to (discrete) loop (L) and tree (T) components $J_{\mathrm{L}}, J_{\mathrm{T}}, M_{\mathrm{L}}, M_{\mathrm{T}}$ of $J$ and $M$. We let $n_{I}$ denote the number of unknowns for component $I$, and set $n:=n_{1}+n_{2}+n_{3}+n_{4}$ (total number of unknowns for the system (3)), so that $Z \in \mathbb{C}^{n, n}, Z^{I J} \in \mathbb{C}^{n_{I}, n_{J}}$ and $X^{I}, Y^{I} \in$ $\mathbb{C}^{n_{I}}$. System (3) is given in detailed form in the Appendix. On using formulas (2) therein, one readily finds that, up to multiplicative dimensional scaling factors, the SIE formulation depends on the physical characteristic parameters only through the non-dimensional numbers $\gamma, \xi$ and $\varepsilon_{r}^{\mathrm{d}}, \mu_{r}$.

\section{A. Global Normalization}

The PMCHWT system (3) can be given the equivalent normalized form

$$
\widetilde{Z} \widetilde{X}=\widetilde{Y} \quad \text { with } \tilde{Z}=N Z N, \tilde{Y}=N Y, X=N \widetilde{X},
$$

with the diagonal normalization matrix $N \in \mathbb{C}^{n, n}$ defined by

$$
N=\operatorname{Diag}\left(\left|Z_{11}\right|^{-1 / 2}, \ldots,\left|Z_{n n}\right|^{-1 / 2}\right) .
$$

The numerical examples of Sec. III will show that system (4) is much better conditioned than the original system (3).

\section{B. Multi-Step Algorithm}

We then propose an alternative approach based on blockwise successive over-relaxation (block SOR) [11]. The idea consists 
in exploiting the $4 \times 4$ block structure of $Z$ given in (3), taking advantage of the fact that the entries of each diagonal submatrix $Z^{I I}$ have the same asymptotic behavior in the limit $\gamma=0$ (while entries of two distinct blocks usually do not), making the submatrices $Z^{I I}$ better conditioned than the global matrix $Z$. Accordingly, we decompose $Z$ according to

$$
Z=L_{\eta}+U_{\eta}
$$

where $L_{\eta}$ and $U_{\eta}$ are lower and upper block-triangular matrices defined by

$$
\begin{aligned}
& {\left[L_{\eta}\right]_{I J}=\left\{\begin{aligned}
Z^{I J} & \text { if } J<I \\
\eta^{-1} Z^{I I} & \text { if } J=I \\
0 & \text { if } J>I
\end{aligned}\right.} \\
& {\left[U_{\eta}\right]_{I J}=\left\{\begin{aligned}
0 & \text { if } J<I \\
(\eta-1) \eta^{-1} Z^{I I} & \text { if } J=I, \\
Z^{I J} & \text { if } J>I
\end{aligned}\right.}
\end{aligned}
$$

in terms of the relaxation parameter $\eta$, which must be chosen such that $\eta \in] 0,2[$ as SOR iterations are known to diverge for $\eta \notin] 0,2[$ [11] (the case $\eta=1$ corresponding to block GaussSeidel iterations). The block SOR algorithm then computes a sequence $\left\{X_{n}\right\}(n \geq 0)$ by recursively solving the systems

$$
L_{\eta} X_{n+1}=Y-U_{\eta} X_{n}
$$

from an arbitrary initial guess $X_{0}$, until the stopping criterion

$$
R_{n}<\epsilon, \quad \text { with } \quad R_{n}:=\left\|X_{n+1}-X_{n}\right\|_{\infty} /\left\|X_{n+1}\right\|_{\infty},
$$

is satisfied. Given the blockwise split (6) of $Z$, each SOR iteration consists in successively solving the four subsystems

$$
\begin{aligned}
Z^{I I} X_{n+1}^{I}= & \eta\left(Y^{I}-\sum_{J>I} Z^{I J} X_{n}^{J}-\sum_{J<I} Z^{I J} X_{n+1}^{J}\right) \\
& -(\eta-1) Z^{I I} X_{n}^{I} . \quad(I=1, \ldots 4)
\end{aligned}
$$

In practice, setting the tolerance to $\epsilon=10^{-3}$ in (8) is sufficient and entails only about 10 SOR iterations for the range of physical parameters typically occurring in electromagnetic testing. When the diagonal blocks $Z^{I I}$ can be held in RAM, we precompute the LU factorization of each $Z^{I I}(I=1 \ldots, 4)$, allowing to reduce each subsequent step of the SOR algorithm to backsubstitutions and matrix-vector products.

The multi-step treatment proposed in [10] for dielectric configurations is based on Gauss-Seidel iterations $(\eta=1)$ for the $2 \times 2$ block partition of the PMCHWT influence matrix induced by the partition of $X$ into two subvectors $X^{1}=\left\{J_{\mathrm{L}}, M_{\mathrm{T}}\right\}$ and $X^{2}=\left\{J_{\mathrm{T}}, M_{\mathrm{L}}\right\}$.

\section{NUMERICAL RESULTS}

In this section, we demonstrate the behavior of the modified systems (4) and (7) through numerical results obtained on three test configurations, focusing on the reduction and stability of the relevant condition numbers, the iteration counts required by the block SOR method, and the influence of the physical regime and the relaxation parameter on its performance. The BE models used here are limited to roughly $10^{4}$ unknowns, allowing to pre-compute LU factorizations in all cases. The definition of non-dimensional parameters $\gamma, \xi, \eta, \epsilon$ is recalled for convenience in Table I.
TABLE I

LIST OF NON-DIMENSIONAL PARAMETERS (WITH $\lambda_{0}$ : WAVELENGTH IN VACUUM AND $\delta$ : SKIN DEPTH) AND THEIR TYPICAL VALUES.

\begin{tabular}{c|ll|}
$\gamma$ & $\gamma:=\sqrt{\omega \varepsilon_{0} / \sigma}=\pi \sqrt{2 \mu_{r}} \delta / \lambda_{0}=o(1)$ & see (1) \\
$\xi$ & $\xi:=L \sqrt{\omega \sigma \mu_{0}}=\sqrt{2} L / \delta=O(1)$ & see (1) \\
$\eta$ & Relaxation parameter of block SOR, $0<\eta<2$ & see (9) \\
$\epsilon$ & Tolerance for stopping rule of block SOR & see (8) \\
\hline
\end{tabular}

TABLE II

SPHERE: VALUES OF $\gamma$ FOR THE CHOSEN PARAMETERS $(f, \sigma)$.

\begin{tabular}{|c|c|c|c|}
\hline$f$ vs $\sigma$ & $10^{-2}$ & $10^{2}$ & $10^{6}$ \\
\hline 1 & $7.45 \times 10^{-5}$ & $7.45 \times 10^{-7}$ & $7.45 \times 10^{-9}$ \\
\hline $10^{3}$ & $2.35 \times 10^{-3}$ & $2.35 \times 10^{-5}$ & $2.35 \times 10^{-7}$ \\
\hline $10^{6}$ & $7.45 \times 10^{-2}$ & $7.45 \times 10^{-4}$ & $7.45 \times 10^{-6}$ \\
\hline $10^{9}$ & 2.35 & $2.35 \times 10^{-2}$ & $2.35 \times 10^{-4}$ \\
\hline
\end{tabular}

\section{A. Conducting sphere}

Our first test example concerns the scattering of a plane wave with frequency $f$ by a sphere (radius $L$, conductivity $\sigma$, $\left.\varepsilon=\varepsilon_{0}, \mu=\mu_{0}\right)$. The sphere surface $\Gamma$ is meshed using 4608 triangular elements, resulting in 13824 unknowns overall (with $n_{1}=n_{3}=2305$ and $n_{2}=n_{4}=4607$, respectively, for the loop and tree components). Several possible values are considered for $(f, \sigma)$, chosen in order to explore the full range $o(1) \leq \gamma \leq$ $O(1)$ (see Table II). For each choice of $(f, \sigma)$, values of the sphere radius $L$ such that $10^{-2} \leq \xi \leq 10^{3}$ are used. We only consider $\xi \leq 1$ for the configuration such that $f=10^{9} \mathrm{~Hz}$ and $\sigma=10^{-2} \mathrm{~S} / \mathrm{m}$, in order to satisfy condition $L \leq \lambda_{0}$ ensuring a frequency low enough for the mesh to remain sufficiently fine relative to the wavelength. Our numerical results for this problem have been validated by comparing the numerical value of the RCS to an analytical reference value based on Mie series (observed relative differences were below 1\%).

For all of the above configurations, and as shown in Fig. 2 for the cases where $5 \times 10^{-2} \leq \xi \leq 10^{2}$ and $(f, \sigma)=\left(1,10^{-2}\right)$ or $\left(10^{9}, 10^{6}\right)$, both the global matrix $Z$ of the normalized system (4) and the matrix blocks $Z^{I I}$ of the block-SOR subsystems (7) were observed to have condition numbers that (a) are far below those of the non-normalized system (3), and (b) do not depend on the parameters $f, \sigma, L$.

In addition, as shown in Table III for several configurations and with the threshold $\epsilon$ of the stopping rule (8) set to $\epsilon=$ $10^{-3}, 10^{-6}$ or $10^{-12}$, the multi-step algorithm converges for $5 \times 10^{-2} \leq \xi \leq 10^{2}$ within a moderate number of iterations that does not depend much on the configuration whenever $L \leq \lambda_{0}$. A similar convergence behavior was moreover found for the multi-step algorithm in cases where $\xi>10^{2}$ and $L \leq \lambda_{0}$ (the corresponding results not being shown because our reference solution is not valid for these configurations). By contrast, convergence could not be achieved for the multi-step algorithm whenever applied to cases for which $\xi \leq 10^{-2}$, a combination that is however outside typical values occurring in applications of electromagnetic testing.

The 2-block GS treatment of [10] requires less iterations than ours when $\xi=1$; for instance, for $(f, \sigma)=\left(1,10^{-2}\right)$ 
TABLE III

SPHERE: SOR ITERATION COUNTS FOR SEVERAL CHOICES OF $(f, \sigma)$, WITH $\epsilon=10^{-3} / 10^{-6} / 10^{-12}, \eta=1$.

\begin{tabular}{|l||c|c|c|c|c|}
\hline$(f, \sigma)$ vs $\xi$ & $510^{-2}$ & $10^{-1}$ & 1 & $10^{1}$ & $10^{2}$ \\
\hline \hline$\left(1,10^{6}\right)$ & $6 / 17 / 36$ & $5 / 9 / 15$ & $5 / 8 / 14$ & $5 / 7 / 12$ & $6 / 9 / 15$ \\
\hline$\left(10^{9}, 10^{6}\right)$ & $6 / 17 / 37$ & $5 / 9 / 15$ & $5 / 8 / 14$ & $5 / 7 / 12$ & $6 / 9 / 15$ \\
\hline$\left(1,10^{-2}\right)$ & $6 / 17 / 36$ & $5 / 9 / 15$ & $5 / 8 / 14$ & $5 / 7 / 12$ & $6 / 9 / 15$ \\
\hline$\left(10^{6}, 10^{-2}\right)$ & $5 / 13 / 33$ & $5 / 9 / 15$ & $5 / 8 / 13$ & $4 / 7 / 12$ & $6 / 12 / 24$ \\
\hline$\left(10^{9}, 10^{-2}\right)$ & $6 / 9 / 16$ & $6 / 9 / 17$ & $19 / 36 / 72$ & $\left(L>\lambda_{0}\right)$ & $\left(L>\lambda_{0}\right)$ \\
\hline
\end{tabular}
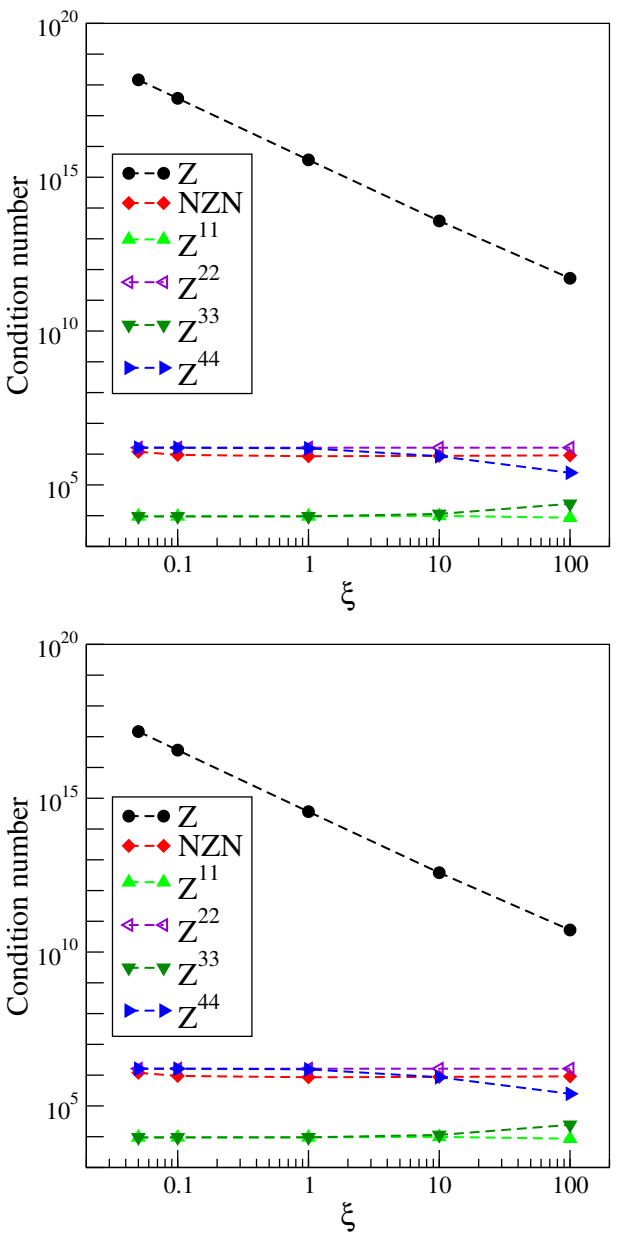

Fig. 2. Sphere: condition numbers obtained for the systems (3) $[\mathrm{Z}]$ and (4) $[\mathrm{NZN}]$ and the sub-systems in (7) $\left[\mathrm{Z}^{11}\right],\left[\mathrm{Z}^{22}\right],\left[\mathrm{Z}^{33}\right],\left[\mathrm{Z}^{44}\right]$, for $(f, \sigma)=$ $\left(1,10^{-2}\right)$ (top) and $\left(10^{9}, 10^{6}\right)$ (bottom).

and a tolerance $\epsilon=10^{-6}$ in (8), it converges at the same rate as our 4-block SOR treatment (17 iterations) for $\xi=5 \times 10^{-2}$, but twice faster ( 4 iterations instead of 9) for $\xi=1$. However, as shown in Fig. 3 and in contrast with Fig. 2, the condition numbers of the relevant diagonal blocks depend strongly on the physical parameters and in particular deteriorate as $\xi$ decreases.

\section{B. Tube}

This example, typical of ECNDT applications in the nuclear power industry, consists of a conducting tube (height $10 \mathrm{~mm}$, internal radius $9.84 \mathrm{~mm}$, thickness $1.27 \mathrm{~mm}, \sigma=10^{7} \mathrm{~S} / \mathrm{m}$ ) excited by an incident field created by a coil (height $2 \mathrm{~mm}$,
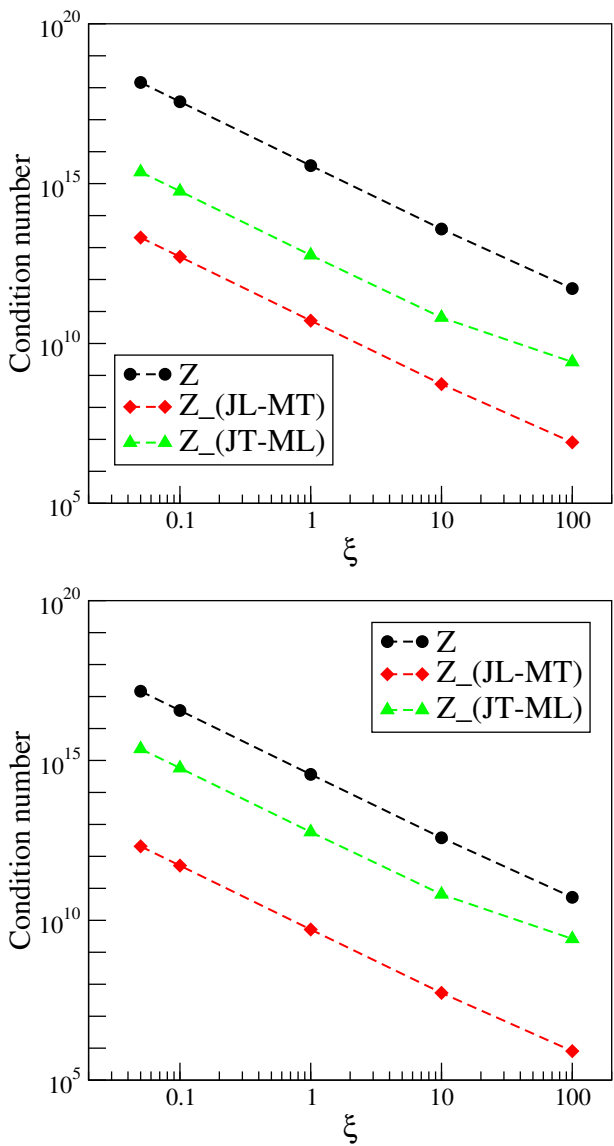

Fig. 3. Sphere: condition numbers obtained for the system (3) $[\mathrm{Z}]$ and the subsystems proposed in [10], with labels [Z_(JL-MT)] and [Z_(JT-ML)] referring to blocks $\left[Z^{11}, Z^{14} ; Z^{41}, Z^{44}\right]$ and $\left[Z^{22}, Z^{23} ; Z^{32}, Z^{33}\right]$, respectively, for $(f, \sigma)=\left(1,10^{-2}\right)$ (top) and $\left(10^{9}, 10^{6}\right)$ (bottom).

internal radius $7.83 \mathrm{~mm}$, thickness $0.67 \mathrm{~mm}, 70$ spires, injected current $1 \mathrm{~A}$ ) whose axis coincides with that of the tube. The tube surface is meshed using 2640 quadrilateral elements (arranged as a regular subdivision of the axial, circumferential and radial coordinates into 20,60 and 2 parts, respectively), and features $10560 \mathrm{BE}$ unknowns $\left(n_{1}=n_{3}=2641, n_{2}=n_{4}=\right.$ 2639). For this topology, the Helmholtz-Hodge decomposition necessitates the addition of two non-local solenoidal basis functions (Fig. 4, [12]). Two cases are considered, the tube being either magnetic $\left(\mu_{r}=100\right)$ or non-magnetic $\left(\mu_{r}=1\right)$.

Numerical solutions for this problem have been computed for the frequency ranges $f \in\left[10^{1}, 10^{3}\right] \mathrm{Hz}$ (magnetic tube) or $f \in\left[10^{3}, 10^{5}\right] \mathrm{Hz}$ (non-magnetic tube). Whereas the respective upper frequency limit corresponds to practical testing 


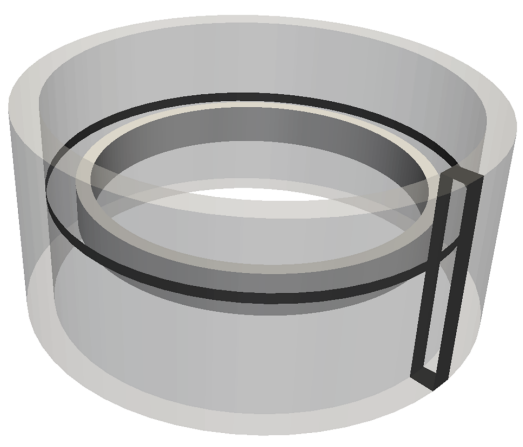

Fig. 4. Tube: schematic showing the coil, the tube segment, and (in black) the support of the two non-local loop basis functions.

conditions, our main goal is to test our block-SOR treatment in the low frequency limit. The observed iteration counts for the block SOR method with tolerance $\epsilon=10^{-6}$ are shown as a function of the relaxation parameter $\eta$ in Fig. 5. For both magnetic and non-magnetic cases, the block SOR method with $0.8 \leq \eta \leq 1$ performs well over a wide frequency range. At lower frequencies, the method is seen to converge only for smaller values of $\eta$, while requiring significantly more iterations, thus gradually becoming inefficient at very low frequencies. Again the range of frequencies in which the block SOR performs well depends on the characteristic size of the conductive body, that corresponds here to the tube thickness, as illustrated in Table IV for a fixed frequency and two values of the tube thickness.

We finally compare our 4-block SOR treatment to the 2block GS treatment of [10], on the configuration defined by $\sigma=10^{7} \mathrm{~S} / \mathrm{m}, \mu_{r}=100$ and for the frequencies $f=$ $1000,600,500 \mathrm{~Hz}$. On the left panel of Fig. 6, the block-GS approach for this problem is seen to converge for $f=1000 \mathrm{~Hz}$ but not for the lower frequencies $f=600,500 \mathrm{~Hz}$. By contrast, the right panel of Fig. 6 shows that appropriate selection of the relaxation parameter (here $\eta=0.8$ ) yields fast convergence for the block-SOR solver applied to the case $f=500 \mathrm{~Hz}$, and that convergence deteriorates and then fails as $\eta$ approaches 1 (the 4-block SOR method coinciding with the 4-block GS method for $\eta=1$ ). The better robustness achieved by the block-SOR
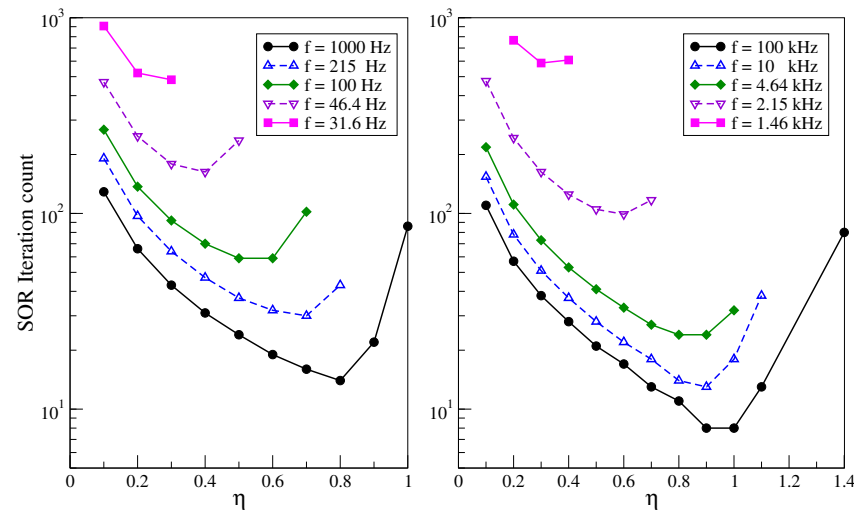

Fig. 5. Tube: iteration count versus the relaxation parameter $\eta$ with stopping criterium $\epsilon=10^{-6}$ for the conducting pipe at various frequencies with $\mu_{r}=$ 100 (left) and $\mu_{r}=1$ (right)
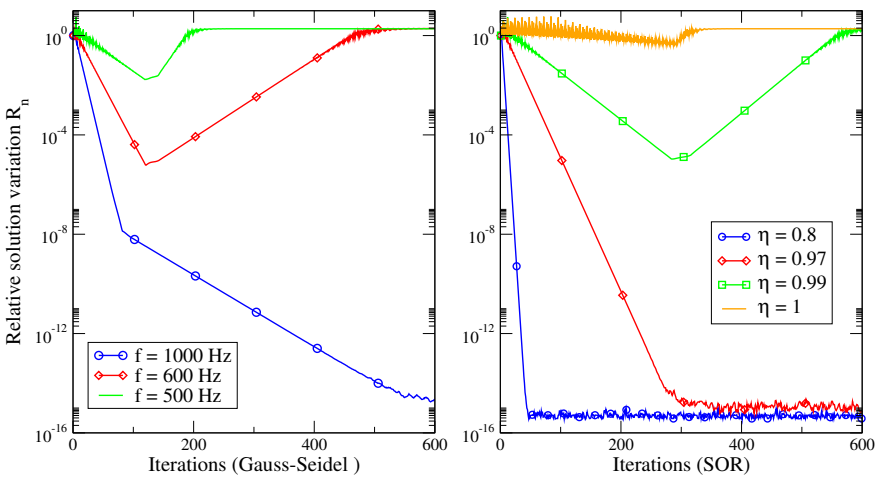

Fig. 6. Tube, $\sigma=10^{7} \mathrm{~S} / \mathrm{m}$ and $\mu_{r}=100$ : convergence behavior of the 2block GS method for $f=1000,600,500 \mathrm{~Hz}$ (left), and of the 4-block SOR method for $f=500 \mathrm{~Hz}$ and varying $\eta$ (right), with $R_{n}$ as defined in (8).

treatment therefore primarily results from the availability (and proper tuning) of the adjustable relaxation parameter $\eta$.

\section{The TEAM-Benchmark Problem No 7}

This last example, which was Testing Electromagnetic Analysis Method (TEAM) benchmark problem \#7, concerns an asymmetrical conducting square plate (conductivity $3.526 \times$ $10^{7} \mathrm{~S} / \mathrm{m}$ ) with a hole. All parameters for this configuration
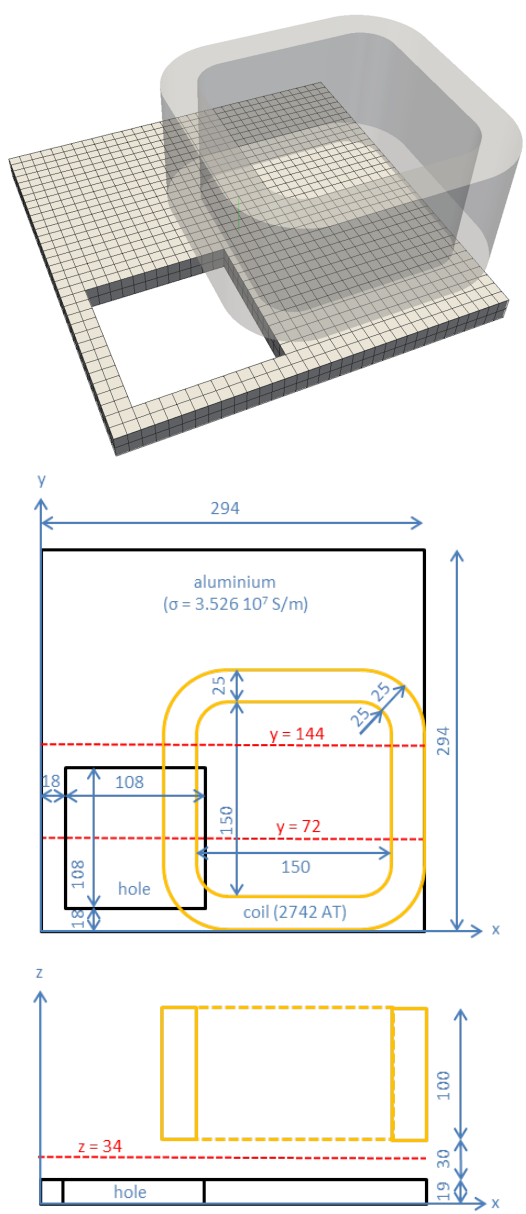

Fig. 7. TEAM benchmark problem \#7: coil geometry (shown in transparency) and mesh (top), geometry, coordinates and dimensions in $\mathrm{mm}$ (center and bottom). 
TABLE IV

TUBE: SOR ITERATION COUNT VERSUS THE RELAXATION PARAMETER $\eta$ WITH $\epsilon=10^{-3}, f=46.4 \mathrm{~Hz}$ AND $\mu_{r}=100$, FOR TWO THICKNESSES $\rho$.

\begin{tabular}{|r||c|c|c|c|c|c|c|c|}
\hline$\eta$ & 0.1 & 0.2 & 0.3 & 0.4 & 0.5 & 0.6 & 0.7 & 0.8 \\
\hline \hline$\rho=1.27 \mathrm{~mm}\left(\xi \simeq 7.7 \times 10^{-2}\right)$ & 486 & 257 & 186 & 171 & 260 & $\times$ & $\times$ & $\times$ \\
\hline$\rho=2.54 \mathrm{~mm}\left(\xi \simeq 1.5 \times 10^{-1}\right)$ & 227 & 116 & 77 & 58 & 47 & 43 & 51 & 452 \\
\hline
\end{tabular}
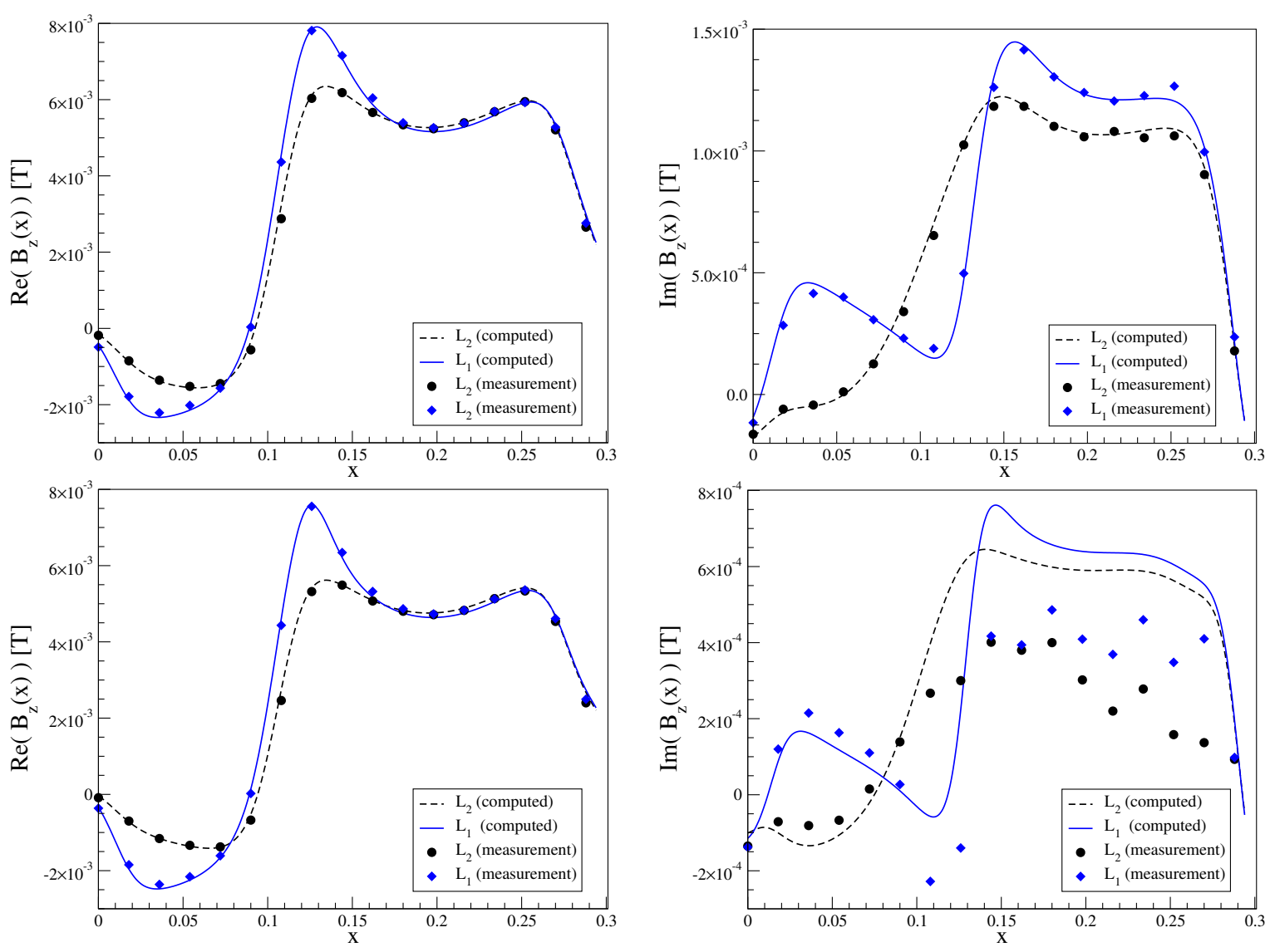

Fig. 8. TEAM benchmark problem \#7: real (left column) and imaginary (right column) parts of the magnetic component $B_{z}$ on the observation lines $L_{1}, L_{2}$ at $50 \mathrm{~Hz}$ (top row) and $200 \mathrm{~Hz}$ (bottom row), with coordinates and dimensions as shown in Fig. 7.

TABLE V

TEAM BENCHMARK PROBLEM \#7: CONDITION NUMBERS AND SOR ITERATION COUNTS, WITH $\epsilon=10^{-3}$ AND $\eta=0.8$.

\begin{tabular}{|r||c|c|c|c|}
\hline Freq. & $\operatorname{cond}(Z)$ & $\operatorname{cond}(\widetilde{Z})$ & $\operatorname{cond}\left(Z^{I I}\right), I=1 \ldots 4$ & SOR iters \\
\hline \hline $50 \mathrm{~Hz}$ & $3.7 \times 10^{20}$ & $1.6 \times 10^{6}$ & $1.4 \times 10^{7} / 3.2 \times 10^{5} / 1.1 \times 10^{7} / 6.1 \times 10^{4}$ & 11 \\
\hline $200 \mathrm{~Hz}$ & $2.8 \times 10^{19}$ & $1.5 \times 10^{6}$ & $1.4 \times 10^{7} / 3.2 \times 10^{5} / 1.1 \times 10^{7} / 3.8 \times 10^{4}$ & 7 \\
\hline
\end{tabular}

are given in $[13,14]$. The quantity of interest is the component $B_{z}(x)$ of the magnetic induction field evaluated between the conductor and the coil generating the incident field, along the two lines $L_{1}$ and $L_{2}$ defined respectively by ( $y=72, z=34$ ) and ( $y=144, z=34$ ), with coordinates and dimensions (in $\mathrm{mm})$ as shown in Fig. 7. Two frequencies are considered: $f=50 \mathrm{~Hz}$ (i.e. $\gamma \simeq 8.9 \times 10^{-9}, \xi \simeq 3.5 \times 10^{1}$ ) and $f=200$ Hz (i.e. $\gamma \simeq 1.8 \times 10^{-8}, \xi \simeq 6.9 \times 10^{1}$ ). The block SOR method is employed with $\eta=0.8$ and a tolerance $\epsilon=10^{-3}$. The BE mesh (Fig. 7) features 2392 quadrilateral elements.

The computed values of $B_{z}(x)$ on $L_{1}$ and $L_{2}$ are seen in Fig. 8 to agree well with experimentally measured values for both chosen frequencies. Moreover, the SOR iteration count and the relevant condition numbers for the three forms (3), (4) and (7) of the discretized PMCHWT system, given in Table V, show that the block SOR approach performs well for both frequencies, reaching convergence within a few iterations.

\section{COMPUTATIONAL CONSIDERATIONS AND OUTLOOK}

The proposed block-SOR multi-step algorithm and the global normalization of the PMCHWT system are both shown to lead to (block or global) matrices whose condition numbers are both acceptable and at worst weakly sensitive to the physical parameters, whereas the original PMCHWT system 
becomes severely ill-conditioned for low $\gamma$, e.g. in the EC regime. This is advantageous on two counts:

(a) Insensitivity to the physical parameters, especially in cases where $\gamma=o(1), \xi=O(1)$ that are our main focus, allows direct application of the PMCHWT formulation of Maxwell's equations, for a wide variety of configurations involving conducting bodies and including EC regimes.

(b) The resulting matrices being well-behaved creates favorable conditions for efficient application of fast BEMs (e.g. the fast multipole method) relying on iterative solvers (e.g. GMRES), should the problem size preclude direct solvers.

The proposed block-SOR treatment also has advantages over solving the global system when both tasks rely on usual direct solvers. Blocks $Z^{J_{A} J_{B}}$ and $Z^{M_{A} M_{B}}$ are symmetric (but not Hermitian) while $Z^{J_{A} M_{B}}$ are skew-symmetric, allowing to set the global systems (3) or (4) in symmetric-indefinite form. Using suitable direct solvers, see [15, Sec. 4.4], this entails $O\left(n^{3} / 3\right)$ and $O\left(n^{2}\right)$ computational work for factoring $Z$ (or $\widetilde{Z})$ and solving resulting triangular systems, respectively, while requiring $O\left(n^{2} / 2\right)$ memory (i.e. roughly half the work and memory needed for LU solvers). By comparison, assuming that the same approximation space is (as here) used for both $J$ and $M$ (so that $n_{1}=n_{3}$ and $n_{2}=n_{4}$ ), the computational work entailed by the block-SOR treatment can be estimated as follows: (a) $O\left(2 n_{1}^{3} / 3+2 n_{2}^{3} / 3\right)$ for factoring the diagonal blocks prior to SOR iterations, (b) $O\left(n^{2}\right)$ per iteration for matrix-vector products (as all blocks are used once each in the matrix-vector products involved in (9) for $I=1, \ldots, 4)$, and (c) $O\left(2 n_{1}^{2}+2 n_{2}^{2}\right)$ per iteration for solving triangular systems (the required memory still being $O\left(n^{2} / 2\right)$ ). For the rooftop quadrangular elements (used in Secs. III-B and III-C), we have $n_{1}=n_{3} \approx n / 4$ and $n_{2}=n_{4} \approx n / 4$, implying $O\left(n^{3} / 48\right)$ and $O\left(n^{2} / 4\right)$ computational work, respectively, for the above tasks (a) and (c). For the RWG triangular elements (used in Sec. III-A), $n_{1}=n_{3} \approx n / 6$ and $n_{2}=n_{4} \approx n / 3$, leading to $O\left(n^{3} / 36\right)$ and $O\left(5 n^{2} / 18\right)$ computational work for tasks (a) and (c). The sixteen- or twelve-fold reduction of the factorization work afforded by the proposed block-SOR treatment is a significant advantage, especially when convergence is reached within modest iteration counts. These considerations are not emphasized here in the numerical examples, as our Matlab coding of the block-SOR solver does not allow to properly exploit symmetries in matrices.

It is sometimes advantageous to still apply direct solvers to large models, e.g. for performing parametric studies on a given physical configuration (for instance, several thousand positions of the exciting coil may be considered in ECNDT or geophysical applications). In this respect, methods combining hierarchical matrices and blockwise low-rank approximation of integral operators [16] are promising by permitting (i) the factorization, with reduced memory and computational complexity, of the resulting compressed approximations of $Z^{I I}$ and (ii) the acceleration of the matrix-vector products involved in (9). Another potentially useful feature of the block SOR approach lies in dissociating the treatment of each component $J_{\mathrm{L}}, J_{\mathrm{T}}, M_{\mathrm{L}}, M_{\mathrm{T}}$ of $J$ and $M$ according to approximations suitable in the regime of interest. A separate curently-ongoing study is devoted to the asymptotic analysis of the integral problem (3) in the limit $\gamma \rightarrow 0$, aiming at a mathematical proof of the fact that the EC model is the limiting form of the Maxwell model as $\gamma \rightarrow 0$.

\section{APPENDIX}

The entries of the influence matrix $Z$ are given, for each block arising from the loop-tree decomposition, by

$$
\begin{aligned}
& Z^{J_{A} J_{B}}=P_{A}\left(\sum_{\ell=0,1} \mathrm{i} \omega \mu_{\ell} S_{A B}^{\ell}\right) P_{B}{ }^{\prime} \\
& Z^{M_{A} M_{B}}=P_{A}\left(\sum_{\ell=0,1} \mathrm{i} \omega \varepsilon_{\ell} S_{A B}^{\ell}\right) P_{B}{ }^{\prime} \\
& Z^{J_{A} M_{B}}=P_{A}\left(\sum_{\ell=0,1} D^{\ell}\right) P_{B}{ }^{\prime}=-Z^{M_{A} J_{B}}
\end{aligned}
$$

where $A, B \in\{L, T\}, P_{L}$ and $P_{T}$ denote the loop and tree projection matrices associated with the discrete approximation space $\operatorname{span}\left(\left\{\boldsymbol{\varphi}_{i}\right\}\right) \subset H_{\mathrm{div}}(\Gamma)$. Moreover, $S^{\ell}$ and $D^{\ell}$ are the discrete boundary integral operators corresponding to Maxwell single- and double-layer potentials for the vacuum $(\ell=0)$ and conducting material $(\ell=1)$, respectively, defined by

$$
\begin{aligned}
{\left[S_{A B}^{\ell}\right]_{t b} } & =\left\langle\boldsymbol{\varphi}_{t}, \boldsymbol{\Psi}^{\ell} \boldsymbol{\varphi}_{b}\right\rangle-\frac{\delta_{A T} \delta_{B T}}{\kappa_{\ell}^{2}}\left\langle\left(\nabla_{\Gamma} \cdot \boldsymbol{\varphi}_{t}\right), \Psi^{\ell}\left(\nabla_{\Gamma} \cdot \boldsymbol{\varphi}_{b}\right)\right\rangle, \\
{\left[D^{\ell}\right]_{t b} } & =\left\langle\boldsymbol{\varphi}_{t}, \nabla \times \boldsymbol{\Psi}^{\ell} \boldsymbol{\varphi}_{b}\right\rangle
\end{aligned}
$$

with $\kappa_{\ell}$ denoting the wave number for medium $\ell$, and

$$
\begin{array}{rlrl}
{\left[\mathbf{\Psi}^{\ell} \mathbf{X}\right](\mathbf{x})} & =\int_{\Gamma} g_{\ell}(\mathbf{x}, \mathbf{y}) \mathbf{X}(\mathbf{y}) \mathrm{d} \mathbf{y} \quad & (\mathbf{x} \in \Gamma), \\
{\left[\Psi^{\ell} X\right](\mathbf{x})} & =\int_{\Gamma} g_{\ell}(\mathbf{x}, \mathbf{y}) X(\mathbf{y}) \mathrm{d} \mathbf{y} \quad(\mathbf{x} \in \Gamma), \\
g_{\ell}(\mathbf{x}, \mathbf{y}) & =\frac{\exp \left(-\mathrm{i} \kappa_{\ell}|\mathbf{x}-\mathbf{y}|\right)}{4 \pi|\mathbf{x}-\mathbf{y}|} &
\end{array}
$$

Finally, the entries in the right-hand side $\{Y\}$ are defined by

$$
\begin{array}{rlrlrl}
Y^{J_{A}} & =P_{A} Y^{J} & \text { with } & & {\left[Y^{J}\right]_{t}} & =\left\langle\boldsymbol{\varphi}_{t}, \mathbf{E}^{\mathrm{inc}}\right\rangle, \\
Y^{M_{A}} & =P_{A} Y^{M} & \text { with } & {\left[Y^{M}\right]_{t}} & =\left\langle\boldsymbol{\varphi}_{t}, \mathbf{H}^{\text {inc }}\right\rangle
\end{array}
$$

where $\mathbf{E}^{\text {inc }}$ and $\mathbf{H}^{\text {inc }}$ are the incident electric and magnetic fields in the vacuum.

\section{ACKNOWLEDGEMENT}

This work has been supported by the CIVAMONT project, aiming at developing scientific collaborations around the NDT simulation platform CIVA developed at CEA LIST.

\section{REFERENCES}

[1] A. Bossavit, Computational Electromagnetism: Variational Formulations, Complementarity, Edge Elements. Academic Press, 1998.

[2] H. Heuer, M. Schulze, M. Pooch, S. Gäbler, A. Nocke, G. Bardl, C. Cherif, M. Klein, R. Kupke, R. Vetter, F. Lenz, M. Kliem, C. Bülow, J. Goyvaerts, T. Mayer, and S. Petrenz, "Review on quality assurance along the CFRP value chain - non-destructive testing of fabrics, preforms and CFRP by HF radio wave techniques," Composites Part B : Engineering, vol. 77, pp. 494-501, 2015.

[3] M. Wilt, H. F. Morrison, A. Becker, H. W. Tseng, K. H. Lee, C. TorresVerdin, and D. Alumbaugh, "Crosshole electromagnetic tomography: A new technology for oil field characterization," The Leading Edge, vol. 14, pp. 173-178, 1994.

[4] W. M. Rucker, R. Hoschek, and K. R. Richter, "Various bem formulations for calculating eddy currents in terms of field variables," Magnetics, IEEE Transactions on, vol. 31, pp. 1336-1341, 1995. 
[5] R. Hiptmair, "Boundary element methods for eddy current computation," in Boundary Element Analysis. Springer, Lecture Notes in Applied and Computational Mechanics, vol. 29, 2007, pp. 213-248.

[6] J.-S. Zhao and W. C. Chew, "Integral equation solution of Maxwell's equations from zero frequency to microwave frequencies," Antennas and Propagation, IEEE Transactions on, vol. 48, pp. 1635-1645, 2000.

[7] S. Y. Chen, W. C. Chew, J. M. Song, and J.-S. Zhao, "Analysis of low frequency scattering from penetrable scatterers," Geoscience and Remote Sensing, IEEE Transactions on, vol. 39, pp. 726-735, 2001.

[8] F. P. Andriulli, "Loop-star and loop-tree decompositions: Analysis and efficient algorithms," Antennas and Propagation, IEEE Transactions on, vol. 60, pp. 2347-2356, 2012.

[9] A. Vigneron, E. Demaldent, and M. Bonnet, "Surface integral equations for electromagnetic testing: The low-frequency and high-contrast case," Magnetics, IEEE Transactions on, vol. 50, pp. 117-120, 2014.

[10] Y. A. Liu and W. C. Chew, "A multi-step iteration scheme to improve the convergence of looptree matrix," Antennas and Propagation Society International Symposium, pp. 1-4, 2008.

[11] R. S. Varga, Matrix iterative analysis. Springer, 2000.

[12] Y. Liu, Y. Chu, and W. C. Chew, "Lffma and induction well-logging modeling," Antennas and Propagation Society International Symposium, pp. 224-227, 2005

[13] "Team workshop: Test problems," 1988.

[14] K. Fujiwara and T. Nakata, "Results for benchmark problem 7," COMPEL, pp. 137-154, 1990.

[15] G. H. Golub and C. F. Van Loan, Matrix computations. Johns Hopkins University Press, Baltimore, 1996.

[16] M. Bebendorf, Hierarchical matrices: a means to efficiently solve elliptic boundary value problems. Springer, Lecture Notes in Computational Science and Engineering, vol. 63, 2008, vol. 63.

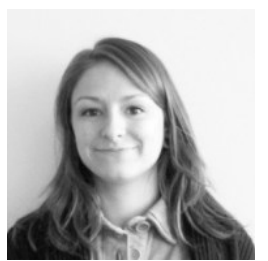

Audrey Vigneron was born in Angers, France, in 1987. She received the M.S. degree in applied mathematics from the University of Rennes 1, Rennes, in 2011 and the Ph.D. degree in applied mathematics at the École Polytechnique in 2015 for her research at CEA (Commissariat à l'Énergie Atomique, France) on integral equations for non destructive electromagnetic testing.

Edouard Demaldent was born in Paris, France, in 1981. He received the $\mathrm{Ph} . \mathrm{D}$. degree in applied mathematics from the University Paris-IX in 2009 for his research at ONERA (Office National d'Études et de Recherches Aérospatial, France) on computational electromagnetics and higher-order methods for RCS. Since then, he is working on numerical methods for the modeling of (mainly electromagnetic) non-destructive testing configurations, at CEA (Commissariat à l'Énergie Atomique, France). His research interests include computational electromagnetics, integral equations and high-order finite element methods.

Marc Bonnet was born in Saverne, France, in 1960. He received his engineer (1983) and doctor (1986) degrees from Ecole Nationale des Ponts et Chaussées (Paris, France), where he studied civil and mechanical engineering, and later (1995) obtained his habilitation from Paris 6 university. He is since 2000 a senior scientist with the French National Center for Scientific Research (CNRS), and was before then (1988-2000) a CNRS junior scientist. He is currently based at the Applied Mathematics department of ENSTA (Palaiseau, France). His research interests include integral equation methods for wave propagation problems, and inverse problems. 